\title{
Densidad poblacional y estructura de talla del pepino de mar Holothuria (Halodeima) grisea (Aspidochirotida: Holothuriidae) en aguas someras del sur del golfo de Morrosquillo, Caribe Colombiano
}

\author{
Carlos Andrés Nisperuza Pérez ${ }^{1}$, Jaminson Padilla Cantero ${ }^{1}$ \& Jorge A. Quirós-Rodríguez ${ }^{1}$ \\ 1. Grupo de Investigación en Biodiversidad Marina y Costera, BIODIMARC, Departamento de Biología, Universidad \\ de Córdoba, Montería, Colombia; carlosbiologia2486@gmail.com, junigon991@hotmail.com, \\ alexander_quiroz@hotmail.com
}

Recibido 19-X-2017. Corregido 11-I-2018. Aceptado 14-II-2018.

\begin{abstract}
Density population and size structure of the sea cucumber Holothuria (Halodeima) grisea (Aspidochirotida: Holothuriidae) in shallow waters south of the gulf of Morrosquillo, Colombian Caribbean. Holothuria grisea, despite not being considered a commercial resource has been the subject of illegal extractions in Colombia, a fact that becomes more serious due to the empty of biological information of these organisms. For this reason the density population and size structure of $H$. grisea were evaluated in three sectors south of the gulf of Morrosquillo from April to November 2015. To this end, three circular transects were located each month in each sampling site covering an area of $300 \mathrm{~m}^{2}$, where individuals were counted and measured in situ. The average density of $H$. grisea in the study area was $0.4 \mathrm{ind}^{-2}$, with the highest values in

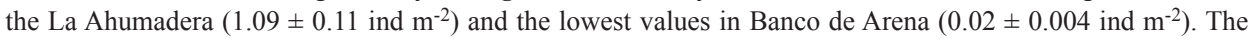
analysis of variance showed a significance in the values of density and size of $H$. grisea in the studied sectors. The species presented a unimodal distribution of sizes with an average size of $13.5 \pm 0.9 \mathrm{~cm}$ with a minimum size of 2.5 and a maximum of $30 \mathrm{~cm}$, intermediate sizes generally showed a higher representativeness throughout this study $(77.2 \%)$. The biological aspects presented in this paper are of great importance for the conservation of this species and corresponds to an advance in the knowledge of the Holothuroidea class in the Colombian Caribbean. Rev. Biol. Trop. 66(2): 776-787. Epub 2018 June 01.
\end{abstract}

Key words: Aspidochirotida; Cispata Bay; echinoderms; marine invertebrate; Thalassia testudinum.

Los holotúridos constituyen una de las cinco clases del filum de los equinodermos, se caracterizan por cumplir un papel importante en la mezcla de los sedimentos y el reciclaje de la materia orgánica en los ecosistemas marinos; estos invertebrados consumen el material orgánico presente en los sedimentos, además lo trituran y transforman en partículas más finas que son devueltas a las capas superiores de los fondos marinos, permitiendo la penetración del oxígeno, lo que los hace importantes en la determinación de la estructura del hábitat para otras especies (Uthicke, 2001).

En Colombia existe un vacío de información sobre la biología y pesquería de holotúridos, por lo tanto, hay un gran potencial para la investigación en diversas áreas relacionadas con estos invertebrados (Forero, Hernández, \& Martinez, 2013). Esto hace evidente la necesidad de elaborar mayores estudios al respecto, con el fin de tener una mejor apreciación de la ecología de este grupo (Quirós-Rodríguez, 2015). En general, los trabajos en holotúridos se han centrado en la realización de inventarios y notas taxonómicas, obviando aspectos poblacionales y reproductivos que son de suma importancia (Ortiz, 2006).

Holothuria (Halodeima) grisea Selenka, 1867, es un pepino de mar del orden Aspidochirotida, caracterizado por presentar un cuerpo subcilíndrico que puede alcanzar $25 \mathrm{~cm}$ de largo aproximadamente (Hendler, Miller, Pawson, \& 
Kierp, 1995). Esta especie habita en praderas de pastos marinos, pero se le puede encontrar en arrecifes y fondos blandos a menos de cinco metros de profundidad (Pawson, Pawson, \& King, 2010). Aunque en Colombia no se habían realizados estudios con $H$. grisea, en las costas brasileñas, ha recibido cierto interés por sus altas abundancias destacándose los trabajos de Mendes, Marenzi, \& Domenico (2006); Martins \& Ventura (2013); De lima Bueno, Garcia, Di Domenico \& Borges (2015) y Leite-Castro et al. (2016) quienes han estudiado aspectos poblacionales como la densidad, distribución espacial y la periodicidad reproductiva.

H. grisea fue reportada por primera vez para el Caribe Colombiano por Álvarez \& Ramon (1981), y pese a que no constituye en la actualidad un recurso comercial, se han registrado pescas ilegales en Santa Marta. En 2009, fueron confiscados 200 individuos (BenavidesSerrato, 2006). En el golfo de Morrosquillo los pescadores han manifestado que en la década de los 90 se presentó una extracción excesiva e insostenible de estos organismos para fines comerciales por parte de empresas orientales, hasta el punto de reducir drásticamente sus poblaciones (E. A. Díaz, comunicación personal, 12 de abril, 2014).

Actualmente, las poblaciones naturales de $H$. grisea, se encuentran sometidas a variaciones intensas en los intercambios fluviales y marinos que se han presentado en el golfo de Morrosquillo, principalmente en el sector sur, como consecuencia de la puesta en funcionamiento de la represa de URRA, aguas arriba del río Sinú (Calle \& Monroy, 2001). De igual forma, el cambio en la desembocadura de este afluente en la década de los 40 , ha provocado una salinización de la bahía de Cispatá, lo que ha incidido drásticamente en la biota del lugar (Sánchez-Páez, Ulloa-Delgado, Tavera-Escobar, \& Gil-Torres, 2005), lo que puede tener cierta influencia acentuada en la estructura poblacional de esta especie, puesto que al ser un holotúrido, puede presentar características intrínsecas como movilidad limitada, madurez tardía, reproducción denso-dependiente, preferencias de hábitat e índices bajos de reclutamiento, lo cual los hacen organismos extremadamente sensibles a las modificaciones ambientales (Uthicke \& Conand, 2005). Debido a la importancia ecológica y el potencial económico que presentan estos organismos, se estimó la densidad poblacional y estructura de talla del pepino de mar $H$. grisea en aguas someras al sur del golfo de Morrosquillo, Caribe Colombiano. Esta información constituye un marco de referencia para proponer medidas de conservación de esta especie y así mitigar los efectos negativos que puedan producirse por una posible explotación a futuro en el área de estudio.

\section{MATERIALES Y MÉTODOS}

Área de estudio: Este trabajo se realizó en el sector sur del golfo de Morrosquillo, Caribe Colombiano, para ello, se realizaron visitas mensuales en tres sectores desde abril hasta noviembre de 2015 (Cuadro 1), con el fin

CUADRO 1

Ubicación geográfica y descripción de los sectores de muestreo en el sur del golfo de Morrosquillo, Caribe Colombiano

TABLE 1

Geographical location and description of sampling points in the south of the gulf of Morrosquillo, Colombian Caribbean

\begin{tabular}{|c|c|c|}
\hline Punto de muestreo & Ubicación geográfica & Descripción \\
\hline Banco de Arena & $09^{\circ} 25^{\prime} 26.15^{\prime \prime} \mathrm{N}$ y $75^{\circ} 47^{\prime} 33.6^{\prime \prime} \mathrm{W}$ & $\begin{array}{l}\text { Sustrato arenoso colonizado por pequeños céspedes } \\
\text { multiespecíficos de algas. }\end{array}$ \\
\hline La Ahumadera & $09^{\circ} 24^{\prime} 51.7^{\prime \prime} \mathrm{N}$ y $75^{\circ} 48^{\prime} 82.1^{\prime \prime} \mathrm{W}$ & $\begin{array}{l}\text { Sustrato fango-arenoso colonizado principalmente por esponjas } \\
\text { y praderas de Thalassia testudinum (Quirós-Rodríguez, 2015). }\end{array}$ \\
\hline Punta Bonita & $09^{\circ} 24^{\prime} 13.9^{\prime \prime} \mathrm{N}$ y $75^{\circ} 48^{\prime} 85.9^{\prime \prime} \mathrm{W}$ & $\begin{array}{l}\text { Sustrato arenoso-calcáreo, con presencia de espolones artificiales } \\
\text { y pequeños parches de Syringodium filiforme. }\end{array}$ \\
\hline
\end{tabular}


de estudiar la posible variación de la densidad de $H$. grisea, teniendo en cuenta el régimen climático. En general, el área de estudio, se caracteriza por ser una región tropical de clima cálido muy húmedo, influenciada por los vientos alisios del noreste y las oscilaciones de la zona de Convergencia intertropical (ZCIT), que regulan las dos principales épocas climáticas que se presentan en el año: el periodo seco que comprende desde mediados de diciembre a abril y el periodo de lluvias, el cual incluye los meses de agosto a noviembre, presentándose las mayores precipitaciones hacia el mes de octubre, los periodos de mayo a julio se consideran como transiciones (Ávila, 2009).

El área de estudio, se caracteriza por tener incidencia directa de la bahía de Cispatá, antigua desembocadura del río Sinú, la geomorfología del litoral indica que es una zona deltaica que ha recibido todos sus sedimentos del río Sinú (Castaño, 2002). Se caracteriza por poseer un sustrato conformado, en su mayoría, por sedimentos finos y muy finos, y algunos depósitos coralinos. Por lo general presenta un microrelieve plano, el cual no supera los diez metros de profundidad (Rojas \& Sierra-Correa, 2010). La temperatura del agua superficial oscila entre 26.5 y $33{ }^{\circ} \mathrm{C}$ y tiende aumentar en dirección a la costa, y la salinidad entre 26.5 y 35 ups, la cual aumenta en dirección contraria a la temperatura (Molina et al., 1994; Gulf, 2004).

Densidad poblacional: Para estimar la densidad poblacional de $H$. grisea en los tres sectores durante abril-noviembre 2015, se consideró la técnica de transectos de barrido circular de $100 \mathrm{~m}^{2}$ propuesta por Richmond \& Martínez (1993), para ello, se utilizó una cuerda de $5.64 \mathrm{~m}$ de longitud (el radio de un círculo de $100 \mathrm{~m}^{2}$ ), la cual se mantuvo fija en un extremo mediante un peso que funcionará como eje de rotación. Uno de los investigadores sujetaba la cuerda en el extremo opuesto al peso, mientras los demás, a partir de buceo SCUBA, recorrían a lo largo de la cuerda buscando y contando todos los individuos que se encuentran dentro del transecto. En cada una de los sitios de muestreo se realizaron tres réplicas aleatoriamente, cubriendo así un área total de $300 \mathrm{~m}^{2}$. La densidad poblacional fue estimada en los sectores de muestreo durante los meses estudiados, empleando la fórmula: $D=N i / A i$. Donde, $N i$ es el número total de individuos por punto y $A i$ es el área total $\left(\mathrm{m}^{2}\right)$ de cada punto muestreado.

Estructura de talla: Se contabilizaron un total de 2766 individuos de $H$. grisea, cada uno de ellos fue medido in situ con una cinta métrica flexible, con el fin de determinar el largo total de los individuos (distancia de la boca al ano), tratando en lo posible de evitar la sobre manipulación de los especímenes, debido a que la mayoría de especies se contraen cuando son estresados, así que, el tamaño corporal puede variar grandemente en relación a animales que no han sido manipulados (Purcell, Lovatelli, Vasconcellos, \& Ye, 2010). Para mayor facilidad las tallas de $H$. grisea se dividieron en tres intervalos teniendo en cuenta la mínima y máxima longitud de los individuos encontrados: pequeños o juveniles $(2.5$ a $11.6 \mathrm{~cm})$, intermedias $(11.7$ a $20.8 \mathrm{~cm})$ y grandes $(20.9$ a $30 \mathrm{~cm}$ ), además se elaboraron histogramas de frecuencias de las tallas mensuales de los organismos encontrados en el área de interés.

Se aplicaron las pruebas de Shapiro-Wilks y Bartlett para determinar si los datos cumplian con los supuestos de normalidad y homocedasticidad (Zar, 1999). Debido a que las variables densidad y talla no presentaron una distribución normal, se realizó una prueba de comparaciones múltiples de Kruskal-Wallis, para determinar si existían diferencias estadísticamente significativas a nivel mensual y por sectores de muestreo para ambas variables. Se aplicó una prueba de comparaciones múltiples de medias de Duncan. Los valores se reportaron como la media \pm error estándar $( \pm \mathrm{SE})$. Esta información se obtuvo mediante el paquete estadístico STATGRAPHIC Centurión XVI y el programa XLSTAT 7.5 (Addinsoft, 2007). 


\section{RESULTADOS}

Densidad poblacional: La densidad media de $H$. grisea al sur del golfo de Morrosquillo durante el estudio, fue de $\overline{\mathrm{X}}=0.4 \pm \mathrm{SE}=0.04$ ind $\mathrm{m}^{-2}$, presentandose los mayores valores en el sector La Ahumadera $\left(1.09 \pm 0.11\right.$ ind $\left.\mathrm{m}^{-2}\right)$, seguida de Punta Bonita $\left(0.07 \pm 0.01\right.$ ind $\left.\mathrm{m}^{-2}\right)$, y por último el sector Banco de Arena (0.02 \pm 0.004 ind $\mathrm{m}^{-2}$ ) (Fig. 1).

La prueba de Kruskal-Wallis registró diferencias estadisticamente significativas en la densidad de $H$. grisea a nivel espacial $(\mathrm{P}<$ 0.05). Al aplicar sobre estos resultados la prueba a posteriori de Duncan, se formaron dos grupos: Los sectores Banco de Arena y Punta Bonita constituyen el grupo A con las medias más bajas, en contraste, el sector La Ahumadera forma el grupo B con la media más alta (Cuadro 2).

La densidad media de $H$. grisea a nivel temporal no registró diferencias marcadas al sur del golfo de Morrosquillo ( $\mathrm{P}>0.05)$. Sin embargo, La Ahumadera presentó las densidades más altas durante el estudio, alcanzando sus máximos valores en los meses de septiembre (mes lluvioso) con 1.51 ind $\mathrm{m}^{2}$. Por el

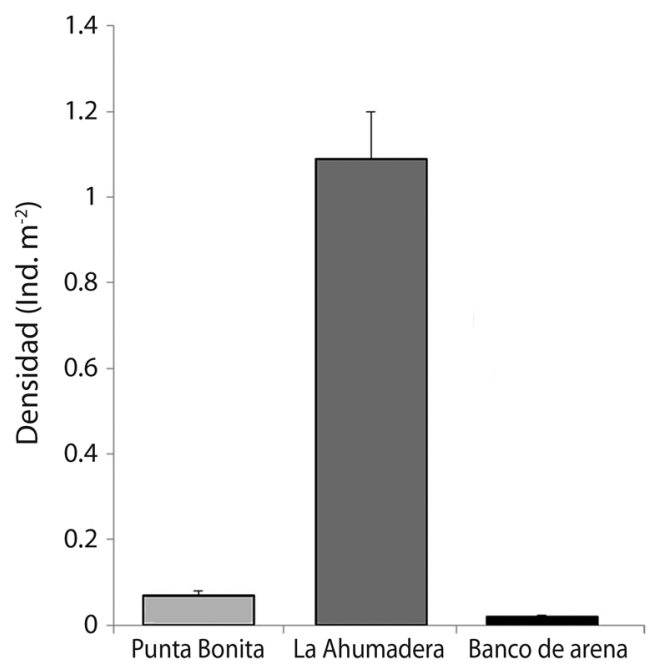

Fig. 1. Densidad media de $H$. grisea en tres sectores del sur del golfo de Morrosquillo.

Fig. 1. Average density of $H$. grisea in three sectors south of the gulf of Morrosquillo.
CUADRO 2

Comparación múltiple de medias de la densidad de H. grisea mediante el procedimiento de Duncan

TABLE 2

Multiple comparison of means of the density of H. grisea by Duncan's procedure

\begin{tabular}{lcc}
\multicolumn{1}{c}{ Sector } & Media estimada & Grupos \\
Banco de Arena & 0.072 & $\mathrm{~A}$ \\
Punta Bonita & 0.075 & $\mathrm{~A}$ \\
La Ahumadera & 1.09 & $\mathrm{~B}$ \\
\hline
\end{tabular}

contrario, durante los meses de abril y mayo, correspondientes al inicio de lluvias esporádicas, se obtuvieron los mínimos valores de densidad $\left(0.01\right.$ ind $\left.\mathrm{m}^{-2}\right)$, ambos registrados para el sector Banco de Arena, es importante destacar que para este sector, durante los meses de junio, julio, septiembre, octubre y noviembre no se registraron individuos; esto mismo sucedió en el sector Punta Bonita durante los meses de junio y julio (Fig. 2).

Estructura de talla: $H$. grisea presentó una distribución de tallas unimodal en el área de estudio, registrándose una talla promedio de $13.5 \pm 0.91 \mathrm{~cm}$, con una mínima reportada de $2.5 \mathrm{~cm}$ y una máxima de $30 \mathrm{~cm}$. La mayor frecuencia de organismos con tallas grandes se presentó en mayo y octubre, correspondiente a meses de transición y lluvias respectivamente. En el caso de individuos con tallas pequeñas, estos fueron más frecuentes durante los meses de abril y septiembre. A lo largo de todo el estudio los organismos con tallas intermedias fueron los más abundantes, con un $77.4 \%$ del total de los individuos registrados (Fig. 3).

El sector La Ahumadera registró mayor predominio de individuos en los tres intervalos de tallas en comparación con los otros sectores estudiados, también fue el sector que obtuvo la talla media más alta $(15.1 \pm 1.23 \mathrm{~cm})$. Por otra parte, la mayoría de los organismos en Punta Bonita presentaron tallas próximas a la media $(13.7 \pm 1.14 \mathrm{~cm})$, mientras que en el sector Banco de Arena se encontraron los individuos con tallas más bajas $(11.7 \pm 0.92$ $\mathrm{cm})$; es necesario resaltar que, debido a la poca 


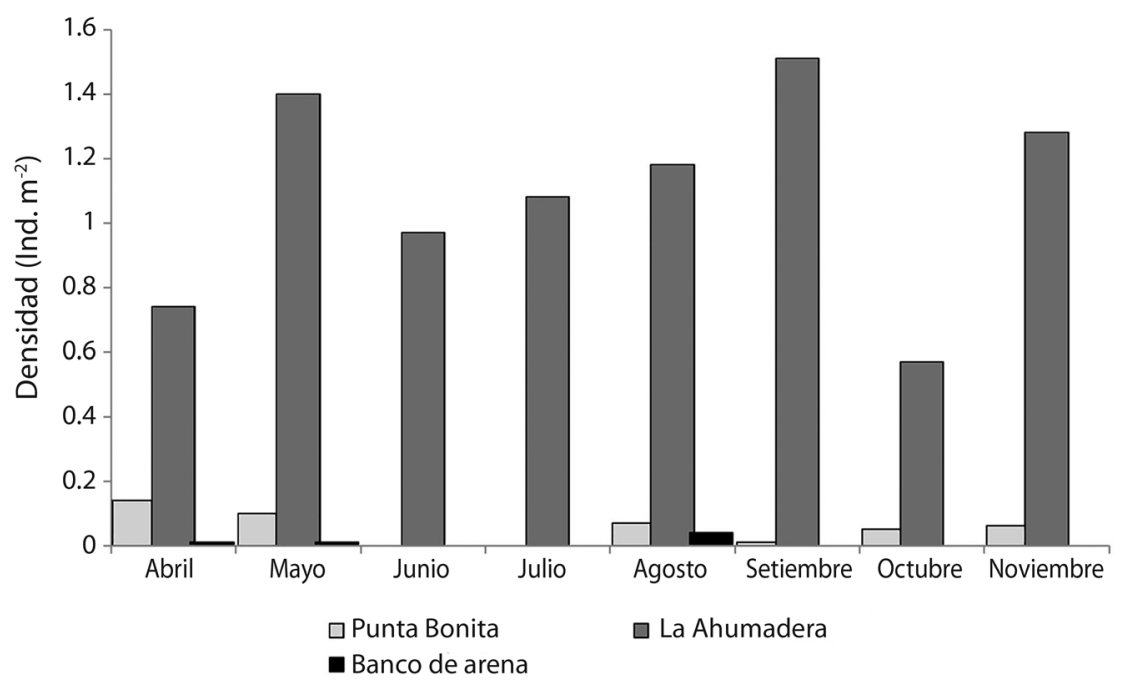

Fig. 2. Comportamiento espacio-temporal de la densidad media de H. grisea en el sur del golfo de Morrosquillo.

Fig. 2. Spatio-temporal behavior of the mean density of H. grisea in the south of the gulf of Morrosquillo.

cantidad de individuos encontrados, no se pudo construir histogramas de frecuencias de tallas para Banco de Arena. Sin embargo, las tallas de los organismos oscilaron entre 10 y $14.9 \mathrm{~cm}$, prevaleciendo individuos pequeños (Fig. 4).

La talla media más alta fue registrada en el mes de octubre en el sector La Ahumadera, hecho que fue casi constante durante todo el periodo de estudio. Por otro lado, la talla media más baja fue reportada en el mes de mayo en el sector Banco de Arena. Los meses sin tallas medias corresponden a aquellos en los cuales, no se encontraron individuos en este estudio (Fig. 5).

CUADRO 3

Comparación múltiple de medias de la talla de H. grisea mediante el procedimiento de Duncan

TABLE 3

Multiple comparison of size averages of $\mathrm{H}$. grisea by Duncan's procedure

\begin{tabular}{lcc}
\multicolumn{1}{c}{ Sector } & Media estimada & Grupos \\
Banco de Arena & 11.7 & $\mathrm{~A}$ \\
Punta Bonita & 13.7 & $\mathrm{~B}$ \\
La Ahumadera & 15.1 & $\mathrm{~B}$ \\
\hline
\end{tabular}

De acuerdo a la prueba de Kruskal-Wallis, se presentaron diferencias estadísticamente significativas en la distribución de tallas de $H$. grisea en los sectores estudiados $(\mathrm{P}<0.05)$. La prueba a posteriori de Duncan demostró la formación de dos grupos; el sector Banco de Arena constituye el grupo A con la media más baja, y los sectores La Ahumadera y Punta Bonita forman el grupo B con las medias más altas (Cuadro 3).

\section{DISCUSIÓN}

Los estuarios, deltas y lagunas costeras sirven como refugio y salacuna de muchos organismos (Sobrino et al., 2005). Tal es el caso de la zona sur del golfo de Morrosquillo, cuya dinámica está estrechamente influenciada por el antiguo delta del río Sinú (Patiño \& Flórez, 1993). Factores como la gran variedad de ecosistemas (pastos marinos, parches arrecifales y bosques de manglar), fondos blandos con vegetaciones mixtas (Quirós-Rodríguez, 2015) y su estado de conservación, pueden explicar la presencia de $H$. grisea en el área de estudio.

El pepino de mar $H$. grisea en esta investigación presentó densidades similares a las 
Abril

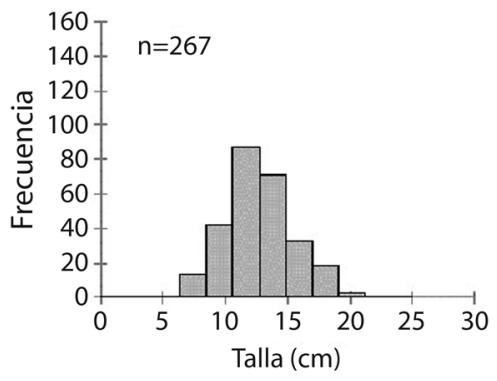

Junio

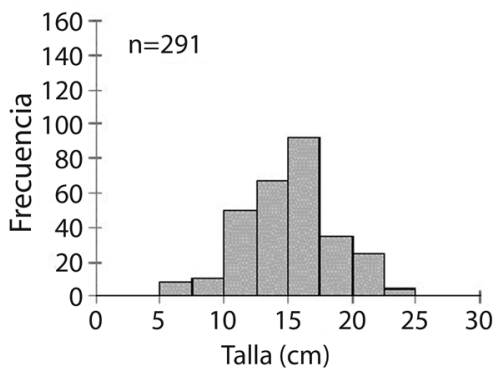

Agosto

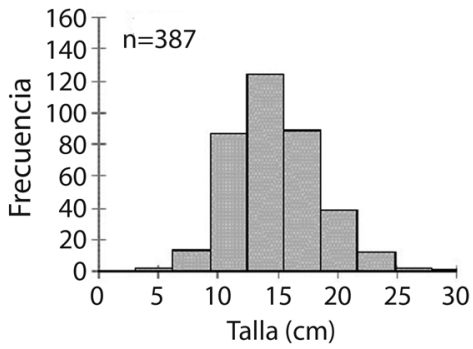

Octubre

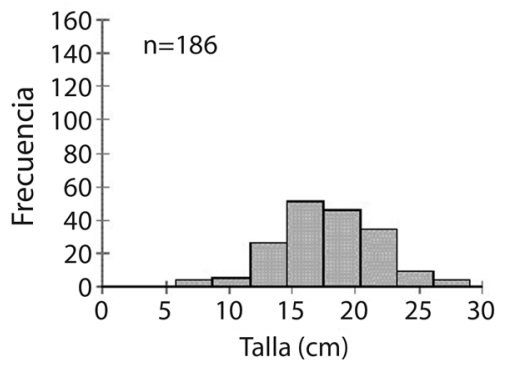

Mayo

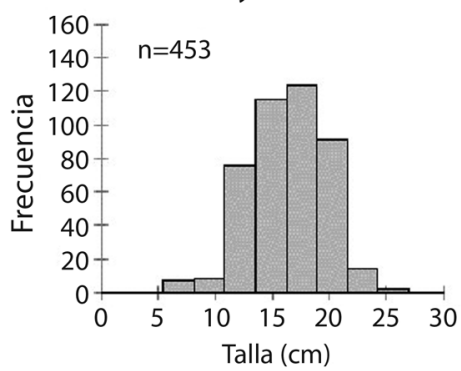

Julio

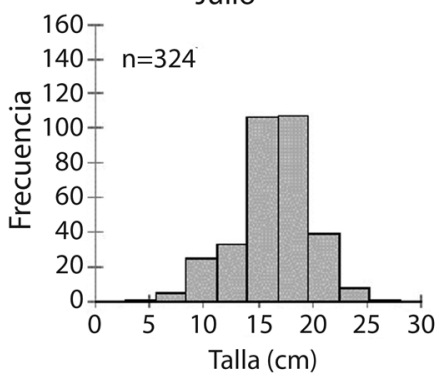

Setiembre
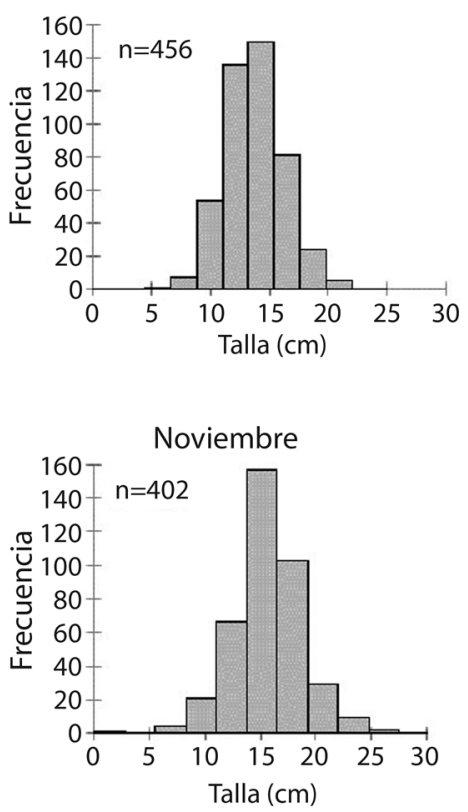

Fig. 3. Frecuencia de tallas registradas para $H$. grisea por mes de muestreo en el sur del golfo de Morrosquillo, Caribe Colombiano.

Fig. 3. Size frequency histogram recorded for $H$. grisea per month of sampling in the south of the gulf of Morrosquillo, Colombian Caribbean. 
A

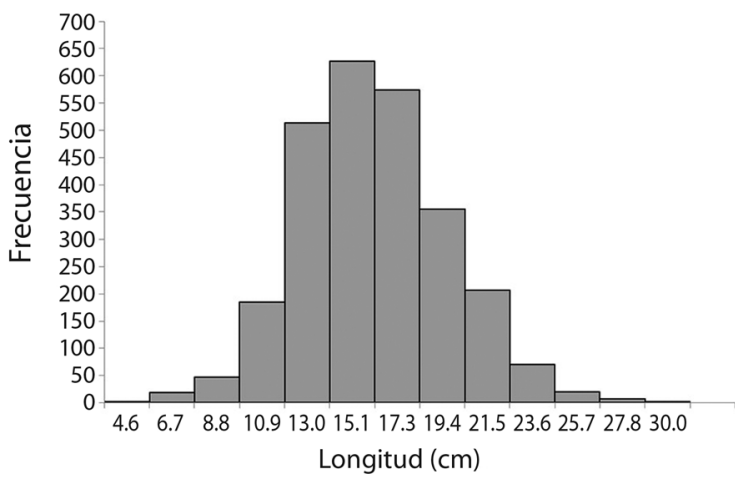

B

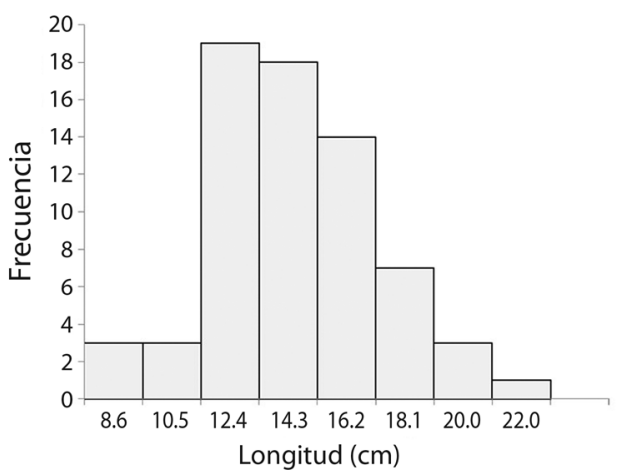

Fig. 4. Distribución de frecuencias de tallas de H. grisea en el sur del golfo de Morrosquillo, a) La Ahumadera, b) Punta Bonita.

Fig. 4. Distribution of size frequencies of H. grisea in the south of the gulf of Morrosquillo, a) La Ahumadera, b) Punta Bonita.

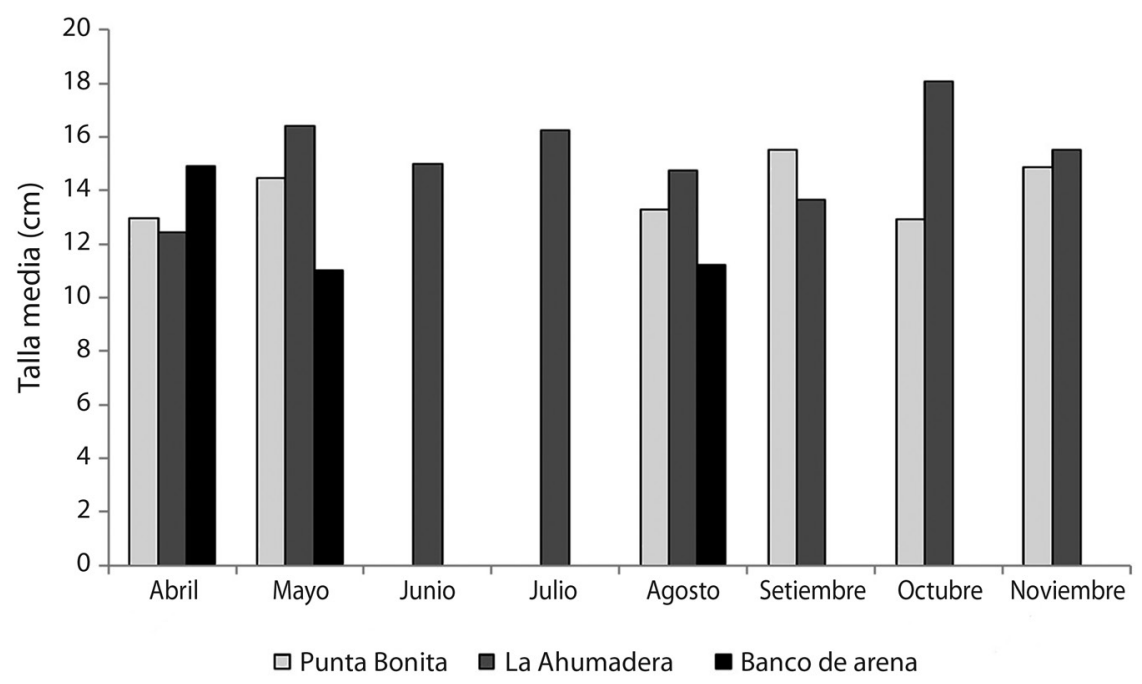

Fig. 5. Comportamiento espacio-temporal de la talla media de H. grisea en el sur del golfo de Morrosquillo.

Fig. 5. Spatio-temporal behavior of the mean size of H. grisea in the south of the gulf of Morrosquillo.

reportadas por Ventura, Verissimo, Nobre \& Zama (2007), en Ilha Grande, y por De Lima Bueno (2010) en Isla Guaratuba, Brasil, quienes registraron para esta especie, densidades medias de 0.3 y 0.15 ind $\mathrm{m}^{-2}$, respectivamente; estas similitudes pueden atribuirse a que el área muestreada, las características del sustrato y la ubicación de los sitios de muestreo (intermareal) fueron similares a los de este estudio. Por el contrario, las densidades obtenidas en este trabajo fueron menores a las registradas por
Méndes, Marenzi \& Domenico (2006) en Santa Catarina-Brasil, con densidades entre 1.60 8.04 ind $\mathrm{m}^{-2}$, hecho que se puede atribuir a que el área muestreada en dicho trabajo comprendía también la zona submareal, sumado a esto, el sustrato presentaba un índice de rugosidad alto, lo cual influye positivamente en la abundancia de holotúridos.

Teniendo en cuenta el potencial pesquero que representa $H$. grisea, los valores de densidad obtenidos en este estudio, son superiores 
CUADRO 4

Densidad y talla de algunas especies de holotúridos de interés comercial

TABLE 4

Density and size of some species of holoturids of commercial interest

\begin{tabular}{|c|c|c|c|c|}
\hline Autor (es) & Localidad & Especie & $\begin{array}{l}\text { Densidad media } \\
\quad \text { (ind } \mathrm{m}^{-2} \text { ) }\end{array}$ & $\begin{array}{l}\text { Talla media } \\
\qquad(\mathrm{cm})\end{array}$ \\
\hline \multirow[t]{2}{*}{ Tagliafico, Rangel \& Rago (2011) } & \multirow[t]{2}{*}{ Isla Cabagua, Venezuela } & Isostichopus badionotus & 0.01 & 18 \\
\hline & & H. mexicana & 0.001 & 16 \\
\hline Aguilar, Revelo, Chacaiza, & Provincia de Santa Elena y & Isostichopus fuscus & 0.03 & 17.3 \\
\hline Mendivez \& Hill (2013) & Sur de Manabí-Ecuador & & & \\
\hline \multirow{2}{*}{$\begin{array}{l}\text { Navarro, García-Sanz } \\
\& \text { Tuya (2013) }\end{array}$} & \multirow[t]{2}{*}{ Islas Canarias, España } & Holothuria. mammata & 0.16 & 23.4 \\
\hline & & H. arguinensis & 0.04 & 25.5 \\
\hline $\begin{array}{l}\text { Dereli, Çulha, Özalp } \\
\text { \& Tekinay (2016) }\end{array}$ & $\begin{array}{l}\text { Estrecho de Dardanelles, } \\
\text { Turquía }\end{array}$ & H. tubulosa & 0.21 & 15.5 \\
\hline $\begin{array}{l}\text { Glockner-Fagetti, Calderom-Aguilera } \\
\text { \& Herrero-Pérezrul (2016) }\end{array}$ & $\begin{array}{l}\text { Reserva Biosfera, Baja } \\
\text { california-México }\end{array}$ & I. fuscus & 0.15 & 17.7 \\
\hline *En esta investigación & $\begin{array}{l}\text { Sur del golfo de } \\
\text { Morrosquillo, Colombia }\end{array}$ & H. grisea & 0.4 & 13.5 \\
\hline
\end{tabular}

a los registrados para otras especies de interés comercial a nivel mundial (Cuadro 4), lo cual puede representar una alternativa viable para el desarrollo de la maricultura a escala futura en el área de interés.

A lo largo del estudio, se determinaron valores de densidad significativamente similares entre Punta Bonita y Banco de Arena, pero en el sector La Ahumadera, se obtuvieron mayores densidades, tendencia que se presentó durante todo el estudio, hecho que se le puede atribuir a la presencia de parches consolidados de Thalassia testudinum, lo cual concuerda con los resultados obtenidos por Conde, Sembrano \& Diaz (1995) y Tagliafico, Rangel \& Rago (2011), quienes encontraron que las especies I. badionotus y H. mexicana, presentaron mayores densidades en zonas donde el sustrato predominante está compuesto por este tipo de pastos marinos en dos localidades de Venezuela. Varios autores han demostrado que la presencia de una alta cobertura de pastos marinos y macroalgas, puede repercutir positivamente en la abundancia de individuos y en la riqueza específica (Alcolado et al., 1998; Valle-García et al., 2008), al generar espacio $\mathrm{y}$ alimento, servir de sustrato de fijación y refugio a otros organismos, y estabilizar los sedimentos (Fitzhardinge, 1983). Asimismo, la ubicación geográfica de La Ahumadera puede ser un factor que favorece la abundancia deorganismos en dicho sector; al ser una zona protegida, en contraste con Punta Bonita y Banco de Arena, se puede permitir el establecimiento de larvas que son transportadas por las corrientes oceanográficas debido a que presentan elevada capacidad de dispersión a través de su rango geográfico (Féral, 2002).

Los resultados obtenidos de la densidad de $H$. grisea en el sector La Ahumadera, también puede tener una explicación biológica compleja que obedece a la existencia de cadenas tróficas en ambientes estuarinos, ya que este sector al tener aporte directo de nutrientes procedentes de la antigua desembocadura del río Sinú, puede propiciar los florecimientos de microalgas, presumiblemente de diatomeas que alimentan a grandes de peces, esta a su vez, alimenta una gran variedad de consumidores terciarios, entre ellos, aves costeras que abundan en estas zonas. Todas las excretas que se producen en estos niveles tróficos, sedimentan 
en forma de materia orgánica que puede ser aprovechada por extensas poblaciones de holoturidos (Solís-Marín et al., 2011).

Los pocos individuos encontrados de $H$. grisea en los sectores Punta Bonita y Banco de Arena, pueden atribuirse a la poca incidencia del antiguo delta del río Sinú en estos sitios, lo cual, puede verse reflejado en un bajo aporte de material orgánico por parte del ecosistema estuarino; los sedimentos que contengan poca materia orgánica estimularían a que los individuos recorran distancias mayores para ocupar sedimentos con contenidos orgánicos más altos (Navarro, García-Sanz, \& Tuya, 2013). Con base en esto, el material arenoso y la poca vegetación que constituye el sustrato, pueden ser factores influyentes en la densidad de individuos en ambos sectores. Tagliafico, Rangel \& Rango (2011) reporta bajas densidades en algunas especies de pepinos de mar en sitios con grandes extensiones de arenales.

La mayor densidad de $H$. grisea registrada en La Ahumadera para el mes de septiembre coincide con la temporada de aguas altas, esto puede favorecer la disponibilidad de nutrientes, debido a la erosión y resuspensión a causa de las mareas, incrementando así, la productividad primaria (Yáñez-Arancibia, Twilley, \& Lara-Dominguez, 1998) y por ende la oferta alimentaria de los pepinos, ya que su dieta, se constituye principalmente de microorganismos, sedimento superficial y detrito (Ruiz, Ibañez, \& Cáceres, 2007). Según Wiedemeyer (1992), la actividad de los holotúridos está fuertemente influida por la abundancia o escases de recursos alimenticios. De acuerdo a Zhou \& Shirley (1996), la distribución espacial de las holotúridos, no es constante durante el año, como se demuestra en la presente investigación, pudiendo existir discrepancias en las abundancias entre meses o incluso días, las cuales pueden estar asociadas a estrategias reproductivas, cambios en la disponibilidad de alimento y condiciones ambientales, entre otros (Conand, 1993).

La talla máxima registrada para $H$. grisea $(30 \mathrm{~cm})$ en el área de estudio fue superior a la indicada por Hendler, Miller, Pawson \&
Kierp (1995), quienes registraron una longitud máxima de $25 \mathrm{~cm}$ para esta especie en la Florida, Estados Unidos. La talla promedio de la especie fue de $13.5 \pm 2.6 \mathrm{~cm}$, ubicándola como una especie pequeña comparada con otras de la familia Holothuriidae y Stichopodidae de interés comercial en varias regiones del mundo (Cuadro 4). En general, las poblaciones de holotúridos están compuestas de individuos de tallas similares (Sloan \& Von-Bodungen, 1980; Laboy-Nieves \& Conde, 2006) como se logró evidenciar en este trabajo, al presentarse predominio de organismos de tallas intermedias durante el estudio. Según Shiell (2007), los individuos jóvenes, son raros de encontrar, o aparecen en poca cantidad, además de presentar reclutamiento bajo y esporádico (Uthicke, Welch, \& Benzie, 2003; Shiell, 2007). Sumado a esto, algunas especies de holotúridos presentan carácter críptico en los primeros estadios de vida, localizándose debajo de rocas y en cavidades poco accesibles (Navarro, García-Sanz, \& Tuya, 2013).

\section{AGRADECIMIENTOS}

Los autores agradecen a la Universidad de Córdoba por el respaldo logístico y financiero; a ASOCAIMAN por todo el apoyo y contribución para la realización de este trabajo y especialmente a Clara Sierra por su interés y aportación al desarrollo del proyecto; a los biólogos José Sáenz Agudelo y Carmen Mercado por su colaboración en el trabajo de campo, y a los 3 revisores anónimos por los comentarios que contribuyeron a mejorar este trabajo.

\section{RESUMEN}

Holothuria grisea, a pesar de no ser considerado un recurso comercial, ha sido objeto de extracciones ilegales en Colombia, hecho que se vuelve más grave debido al vació de información biológica de estos organismos. Por esta razón, se estimó la densidad poblacional y estructura de talla de $H$. grisea en tres sectores del sur del golfo de Morrosquillo de abril a noviembre 2015. Para ello, se ubicaron mensualmente tres transectos circulares en cada sector de muestreo abarcando un área de $300 \mathrm{~m}^{2}$, donde se contabilizaron y midieron in situ los individuos 
encontrados. La densidad media de $H$. grisea en el área estudiada fue 0.4 ind $\mathrm{m}^{-2}$, presentándose los mayores valores en el sector La Ahumadera $\left(1.09 \pm 0.11\right.$ ind $\left.\mathrm{m}^{-2}\right)$ y los menores valores en el Banco de Arena (0.02 \pm 0.004 ind $\mathrm{m}^{-2}$ ). El análisis de varianza señalo una diferencia significativa en los valores de densidad y talla de $H$. grisea en los sectores estudiados. La especie presentó una distribución de tallas unimodal, registrándose una talla promedio de $13.5 \pm 0.91 \mathrm{~cm}$ con una talla mínima de 2.5 y una máxima de $30 \mathrm{~cm}$, en general las tallas intermedias presentaron una mayor representatividad a lo largo de este estudio (77.2\%). Los aspectos biológicos presentados en este documento son de gran importancia para la conservación de esta especie y corresponde a un avance en el conocimiento de la clase Holothuroidea en el Caribe Colombiano.

Palabras clave: Aspidochirotida; Bahía de Cispatá; equinodermos; invertebrado marino; Thalassia testudinum.

\section{REFERENCIAS}

Addindoft. (2007). XLASTAT, Analyse de donées et statistique avec MS [Computer softare]. NY, USA: Addindoft.

Aguilar, F., Revelo, W., Chacaiza, D., Mendivez, W., \& Hill, D. (2013). Distribución y densidad poblacional del pepino de mar Isostichopus fuscus (Ludwig, 1875) en la provincia de Santa Elena y sur de Manabí. Boletín Científico y Técnico, 23(1),73-98.

Alcolado, P., Espinosa, J., Martínez-Estalella, N., Ibarzabal, D., Del Valle, R., \& Martínez-Iglesias, J. (1998). Prospección del megazoobentos de los fondos blandos del Archipiélago Sabana-Camagüey, Cuba. Avicennia, 1(8/9),87-104.

Álvarez, L. R., \& Ramón, L. (1981). Listado preliminar de los equinodermos de la costa Atlántica colombiana. Boletín Museo del Mar, 10, 24-39.

Ávila, V. (2009). Estructura de la taxocenosis MolluscaCrustacea-Echinodermata asociada a los fondos blandos de dos años diferentes (Trabajo de grado para optar al título de Biólogo marino). Universidad Jorge Tadeo Lozano, sede Caribe, Santa Marta, Colombia.

Benavides-Serrato, M. (2006). Taxonomic list of the shallow water echinoderms of Puerto Rico with new information for La Parguera (Tesis de Maestría). Universidad de Puerto Rico, Mayagüez, Puerto Rico.

Castaño, C. (2002). Golfos y bahías de Colombia. Bogota D.C., Colombia: Editorial Banco de Occidente.

Calle, G. M., \& Monroy, G. G. (2001). Ecologia y efecto ambiental de embalses: aproximación con casos colombianos. Universidad Nacional de Colombia, Bogota, D.C., Colombia: Editorial Monica del Pilar Rada Tabón.
Conand, C. (1993). Reproductive biology of the holothurians from the major communities of the New Caledonian Lagoon. Marine Biology, 116, 439-450.

Conde, J. E., Sambrano, A., \& Díaz, H. (1995). Sedimertivorous activity, density and spatial distribution of the deposit-feeding holothurians Holothuria Mexicana Ludwing and Isostichopus badionotus Selenka. Manuscrito, Caracas, Distrito Federal, Venezuela.

De Lima Bueno, M., Garcia Tavares, Y. A., Di Domenico, M., \& Borges, M. (2015). Gametogenesis and weight change of body organs of the sea cucumber Holothuria (Halodeima) grisea (Aspidochirotida: Holothuriidae) in Southern Brazil. Revista de Biología Tropical, 63(2), 285-296.

De lima Bueno, M. (2010). Biología reproductiva de Holothuria grisea Selenka, 1867 (Echinodermata: Holothuroidea) em Guaratuba-Paraná-Brasil (Trabalho de Conclusão de Curso apresentado como requisito para a conclusão do curso de Ciências Biológicas) Faculdade Estadual de Filosofia, Ciências e Letras de Paranagua, Brasil.

Dereli, H., Çulha, S., Culha, M., Özalp, B., \& Tekinay, A. (2016). Reproduction and population structure of the sea cucumber Holothuria tubulosa in the Dardanelles Strait, Turkey. Mediterranean Marine Science, 17(1), 47-55.

Féral, J. (2002). How useful are the genetic markers in attempts to understand and manage marine biodiversity? Journal of Experimental Marine Biology Ecology, 268, 121-145.

Fitzhardinge, R. (1983). Comparisons of the invertebrate faunas colonizing soft sediments in two different habitats. Bulletin of Marine Science, 33(3), 745-752.

Forero, A. R., Hernández, W. V., \& Martinez, V. A. (2013). First insight into Colombian Caribbean sea cucumbers and sea cucumber fishery. SPC Beche-de-Mer Information Bulletin, 33, 9-13.

Glockner-Fagetti, A., Calderon-Aguilera, L., \& HerreroPérezrul, M. (2016). Density decrease in an exploited population of brown sea cucumber Isostichopus fuscus in a biosphere reserve from the Baja California peninsula, Mexico. Ocean \& Coastal Management, $121,49-59$.

Gulf, M. (2004). Dinámica temporal de la asociación de peces en dos arrecifes artificiales del golfo de Morrosquillo, Caribe colombiano. Revista Actualidades Biológicas, 26 (81), 219-230.

Hendler, G., Miller, J., Pawson, D., \& Kierp, P. (1995). Sea stars, sea urchins, and allies Echinoderm of Florida and the Caribbean. Washintong: Smithsonian Institution Press.

Laboy-Nieves, E. N., \& Conde, J. E. (2006). A new approach for measuring Holothuria mexicana and 
Isostichopus badionotus for stock assessment. SPC Beche-de-mer information Bulletin, 24, 39-44.

Leite-Castro, L. V., de Souza Junior, J., Salmito-Vanderley, C. S. B., Nunes, J. F., Hamel, J. F., \& Mercier, A. (2016). Reproductive biology of the sea cucumber Holothuria grisea in Brazil: importance of social and environmental factors in breeding coordination. Marine Biology, 163(3), 1-13.

Martins, A., \& Ventura, C. (2013). Ciclo gametônico do pepino-do-mar Holothuria (Halodeima) grisea (Echinodermata: Holothuroidea). Proceedings of the 2th Latin American Congress of Echinoderms, 61.

Méndes, F., Marenzi, A., \& Domenico, M. (2006). Population patterns and seasonal observations on density and distribution of Holothuria grisea (Holothuroidea: Aspidochirotida) on the Santa Catarina Coast,. Bulletin Becher-de-Mer, 23, 5-10.

Molina, A., Molina, C., Giraldo, L., Parra, C., \& Chevillot, P. (1994). Dinámica marina y sus efectos sobre la geomorfología del golfo de Morrosquillo. Boletín Cientifico del CIOH, 15, 93-113.

Navarro, P. G., García-Sanz, S., \& Tuya, F. (2013). Patrones de abundancia y talla de Holothuria sanctori, Holothuria mammata y Holothuria arguinensis (Echinodermata: Holoturoidea) en la isla de Gran Canaria, Atlántico oriental. Revista de Biología Marina y Oceanografía, 48(2), 273-284.

Ortiz, E. (2006). Cambio espicular en algunos pepinos de mar del genero Holothuria Linnaeus, 1758 (Echinodermata: Holothuroidea) presentes en el Caribe y Pacifico Colombiano (Trabajo de grado para optar al titulo de Biólogo marino). Universidad Jorge Tardeo Lozano, Bogota, Colombia

Patiño, F., \& Flórez, F. (1993). Estudio ecológico del golfo de Morrosquillo (1 ed.). Bogotá, Colombia: Editorial Universidad Nacional de Colombia.

Pawson, D. L., Pawson, D. J., \& King, R. A. (2010). A taxonomic guide to the Echinodermata of the South Atlantic Bight, USA: 1. Sea cucumbers (Echinodermata: Holothuroidea). Zootaxa, 2449, 1-48.

Purcell, S. W., Lovatelli, A., Vasconcellos, M., \& Ye, Y. (2010). Manejo de las pesquerías de pepino de mar con un enfoque ecosistémico. Roma, Italia: FAO Fisheries and Aquaculture

Quirós-Rodriguez, J. A. (2015). Equinodermos en fondos someros del sector La Ahumadera, Bahia de Cispatá, Córdoba, Caribe Colombiano. Acta Biologica Colombiana, 20(1),101-108.

Richmond, R., \& Martínez, P. C. (1993). Sea cucumber fisheries in the Galápagos islands: Biological aspects, impacts and concerns (Informe Técnico). Submitted to the World Conservation Union (IUCN).
Rojas, X., \& Sierra-Correa, P. (2010). Plan integral de Manejo del Distrito de Manejo Integrado (DMI) bahía de Cispatá-La Balsa-Tinajones y sectores aledaños del delta estuarino del río Sinú, departamento de Córdoba. Santa Marta, Colombia: Editorial INVEMAR.

Ruiz, J., Ibáñez, C. M., \& Cáceres, C. W. (2007). Morfometría del tubo digestivo y alimentación del pepino de mar Athyonidium chilensis (Semper, 1868)(Echinodermata: Holothuroidea). Revista de Biología Marina y Oceanografia, 42(3), 269-274.

Sánchez-Páez, H., Ulloa-Delgado, G., Tavera-Escobar, H., \& Gil-Torres, W. (2005). Plan de manejo integral de los manglares de la zona de uso sostenible sector estuarino de la bahía de Cispatá departamento de Córdoba. Bogota D.C, Colombia: Corporación Autónoma Regional de los Valles del Sinu y San Jorge (CVS). Corporación Nacional de Investigaciones y Fomento forestal (OIMT).

Sobrino, I., Baldó, F., García-González, G., Cuesta, A., Silva-García, C., Fernández-Delgado, A., Arias, M., Rodríguez, A., \& Drake, P. (2005). The effect of estuarine fisheries on juvenile fish observed within the Guadalquivir Estuary (SW Spain). Fisheries Research, 76 (2), 229-242.

Shiell, G. (2007). Spatial distribution and temporal shifts in the biology of Holothuria whitmaei Bell Ningaloo Reef, Western Australia (PhD thesis). La Trobe University, Wodinga. Recovered from: http:/www.spc. int/DigitalLibrary/Doc/FAME/InfoBull/BDM/26/ BDM26_35_Shiell.pdf

Sloan, N., \& Von-Bodungen, B. (1980). Distribution and feeding of the sea cucumber Isostichopus badionotus in relation to shelter and sediment criteria of the Bermuda platform. Marine Ecology Progress Series, $2,257-264$

Solís-Marin, F. A., Madrigal, X., Honey-Escandón, M.B, Arriaga-Ochoa, J. A., Caballero, A., García, A., \& Salazar, P. (2011). Diagnóstico de las poblaciones arrecifales del pepino de mar Holothuria inornata Semper, 1868 en la costa del estado de Michocan. Michoacan, Mexico: Secretaria de medio ambiente y recursos naturales.

Tagliafico, A., Rangel, M. S., \& Rago, N. (2011). Distribución y densidad de dos especies de holoturoideos en la isla de Cubagua, Venezuela. Revista de Biología Tropical, 59(2), 843-852.

Uthicke, S. (2001). Interactions between sediment-feeders and microalgae on coral reefs: Grazing losses versus production enhancement. Marine Ecology Progress Series, 210, 125-138. http://doi.org/10.3354/ meps 210125

Uthicke, S., Welch, D., \& Benzie, J. (2003). Slow growth and lack of recovery in overfished holothurians on the Great Barrier Reef: evidence from DNA fingerprints 
and repeated large-scale surveys. Conservation Biology, 18, 1395-1404

Uthicke, S., \& Conand, C. (2005). Local examples of beche-de-mer overfishing: An initial summary and request for information. SPC beche-de-mer Information Bulletin, 21, 9-14.

Valle-García, R. D., Abreu Pérez, M., Rodríguez, R., SolísMarín, F. A., Laguarda-Figueras, A., \& Duran Gonzalez, A. (2008). Equinodermos (Echinodermata) del occidente del Archipiélago Sabana-Camagüey, Cuba. Revista de Biologia Tropical, 56(3), 19-35

Ventura, C. R, Verissimo, I., Nobre, C. C., \& Zama, P.C. (2007). Filo Echinodermata. In H. Passeri- Lavrado \& M. Sá Viana (Eds.), Atlas de invertebrados marinhos da região central da Zona Econômica Exclusiva brasileira (pp. 218-258). Rio de Janeiro, Brasil: Museu Rio de Janeiro.
Wiedemeyer, W. (1992). Feeding behaviour of two tropical holothurians Holothuria (Metriatyla) scabra (Jäger, 1833) and H. (Halodeima) atra (Jäger, 1833), from Okinawa, Japan. In R. H. Richmond (Ed.), Proceedings of the Seventh International Coral Reef Symposium, Guam, 2, 854-860.

Yáñez-Arancibia, A., Twilley, R. R., \& Lara-Domínguez, A. L. (1998). Los ecosistemas de manglar frente al cambio climático global. Madera y Bosques, 4(2), $3-19$.

Zar, J. (1999). Biostatiscal Analysis. New Jersey, USA: Prentice Hall.

Zhou, S. \& Shirley, T. (1996). Habitat and depth distribution of the red sea cucumber Parastichopus californicus in a Southeast Alaska bay. Alaska. Fishery Research Bulletin, 3(2), 123-131. 